\title{
ALL SYMBOLS WORD GENERATED BY CFG
}

\author{
Thinh D. Nguyen \\ Email: kosmofarmer@yandex.com
}

\begin{abstract}
We show that it is NP-complete to determine if a given contextfree grammar can generate a word containing all symbols of its alphabet with each symbol only once.
\end{abstract}

\section{Problem STATEMENT}

Our problem is: given a context free grammar $\mathbf{G}$, check if $\mathbf{G}$ generates a word containing all symbols from its alphabet with each symbol occurring only once.

This problem should be understood as a language over finite alphabet, although alphabet of $\mathbf{G}$ can be arbitrarily big. We assume that symbols of this alphabet are encoded as binary strings.

\section{PROOF OF HARDNESS}

The remark from previous section addresses the following problem: How to encode context-free grammars over an arbitrary alphabet using a fixed alphabet?

Besides the alphabet, we encounter a similar problem when trying to represent non-terminals. We choose to use binary encoding. For example, the grammar

$$
\begin{aligned}
\mathbf{S} & \rightarrow \mathbf{S A} \mid \epsilon \\
\mathbf{A} & \rightarrow a|b| c
\end{aligned}
$$

could be encoded as follows:

$$
\begin{gathered}
\mathbf{N} 0 ; \rightarrow \mathbf{N} 0 ; \mathbf{N} 1 ; \mid \epsilon \\
\mathbf{N} 1 ; \rightarrow \mathbf{T} 0 ;|\mathbf{T} 1 ;| \mathbf{T} 10 ;
\end{gathered}
$$

Having been able to encode an arbitrary context-free grammar, we show the hardness of our problem by reducing from $\mathbf{X 3 C}$. We have a start non-terminal. We add right-recursive grammar rules to be able to generate an arbitrary (ordered) subset of 3-sets, where each 3-set is represented as a non-terminal. Then, for each 3-set, we add the grammar rule to deduce its 3 contained elements of the universe set. Then it is obvious that our grammar can generate a word required by our problem if and only if the original X3C instance possesses an exact cover.

\section{CONCLUS ION}

Garey and Johnson [1] shape their theory based on previous primal works of Cook, Levin and Karp. Johnson [2] moves on with the guide to this theory. As long as we study a mathematical conjecture, we should encourage ourselves to have moderate amount of labour hours on popular mathematics books like these. Then, reading some articles on theory of computing like [3] is a good practice. Having all those done, we could think of the ultimate final for all mathematics sciences.

\section{REFERENCE}

1. Michael R. Garey, David S. Johnson, Computers and Intractability: A Guide to the Theory of NP-Completeness 
2. David S. Johnson, The NP-Comple teness Column: An Ongoing Guide

3. Phan Dinh Dieu, Le Cong Thanh, Le Tuan Hoa, Average Polynomial Time Complexity of Some NP-Complete Problems, Theor. Comput. Sci. 46(3): 219-237 (1986) 